\title{
Two-layer Integrated Optical Tapped Delay Line for RF Spectrum Analysis
}

\author{
J.R. Adleman ${ }^{1}$, C.L. Lin ${ }^{1}$, B.M.L. Pascoguin ${ }^{1}$, B. Neuner III $^{1}$, A. Hening ${ }^{1}$, M. Lasher ${ }^{1}$, E.W. Jacobs ${ }^{1}$ and J.S. \\ Rodgers $^{2}$ \\ 1. SPAWAR Systems Center Pacific,, 53560 Hull St. San Diego, CA 92152. \\ 2. DARPA Microsystems Technology Office, 675 N. Randolph St., Arlington, VA 22203 \\ james.adleman@navy.mil
}

\begin{abstract}
We present the design and characterization of tapped delay line filters fabricated in twolevel photonic integrated circuits. Phase error correction techniques are evaluated to enable spectrum analysis of a $4 \mathrm{GHz}$ band with $300 \mathrm{MHz}$ resolution.

Keywords: Microwave Photonics; Integrated Optics.
\end{abstract}

\section{Introduction}

Development of microwave communications and radar systems has spurred interest in photonic domain approaches for high instantaneous bandwidth (IBW) spectrum analysis (SA) of wideband RF signals. Optical discrete time filters have been considered to perform demultiplexing and channelization of microwave signals [1]. In particular, optical phased array devices such as arrayed waveguide gratings (AWGs) have been exploited successfully in telecommunications to demultiplex channels $25-100 \mathrm{GHz}$ wide. However, these devices are not feasible for channel resolution bandwidths (RBW) below a few $\mathrm{GHz}$, due to their physical scaling requirements.

Previously we presented a design for a photonic integrated circuit (PIC) filter based on an optical phased array fed by a tapped delay line [2]. This approach allows much longer tap delays in a smaller footprint than conventional AWGs, enabling a filter $\mathrm{RBW}<1 \mathrm{GHz}$ to be implemented within a few square centimeters. In this paper we will describe characterization of time and frequency response of fabricated delay line circuits designed for $300 \mathrm{MHz} \mathrm{RBW}$.

\section{Design}

In a phased array, the number and positions of input and output waveguides are chosen based on desired channel spacing, channel width (i.e. RBW), and free spectral range (FSR) [3]. Sufficient delay length, $\Delta L$, must be chosen to separate channel center frequencies to match the output spacing, while channel resolution matching the output waveguide size depends on the number of taps, $N$, that make up the input array. As shown in figure 1 , our design uses two layers of buried channel waveguides: the lower layer contains a spiral delay line; the upper layer contains the tap waveguides and the phased array lens. Optical propagation loss, $\alpha$, in the PIC limits $N$. Since the exact waveguide loss was not known before fabrication we designed multiple delay line filters on a six inch wafer, covering $\alpha=1,2$, and 5 $\mathrm{dB} / \mathrm{m}$, which limits $N<50$. Optimized phased array designs were chosen with $N=40$, which allow RBW of $300 \mathrm{MHz}$ with IBW of $4.2 \mathrm{GHz}$.

The two waveguide layers are separated by an intermediate cladding allowing directional coupling between the spiral and tap waveguides. Tap coupling ratios are set by the crossing angle of the waveguides, making the ratios insensitive to small translational alignment errors. We performed simulations of the crossing couplers using a commercial beam propagation method solver (RSOFT BeamProp) and determined that a intermediate cladding of $4.3 \mu \mathrm{m}$ allows for coupling ratios from 0 to $-30 \mathrm{~dB}$ using angles between 0.25 and 4 degrees. We select tap weights to produce a Taylor windowed power distribution (Figure 1(B) ) at the phased array input in order to reduce filter sidelobes below $25 \mathrm{~dB}$. Coupling ratios are determined via a recurrence relation working back from the $N^{\text {th }}$ tap, using waveguide propagation loss and the target tap weights as parameters.
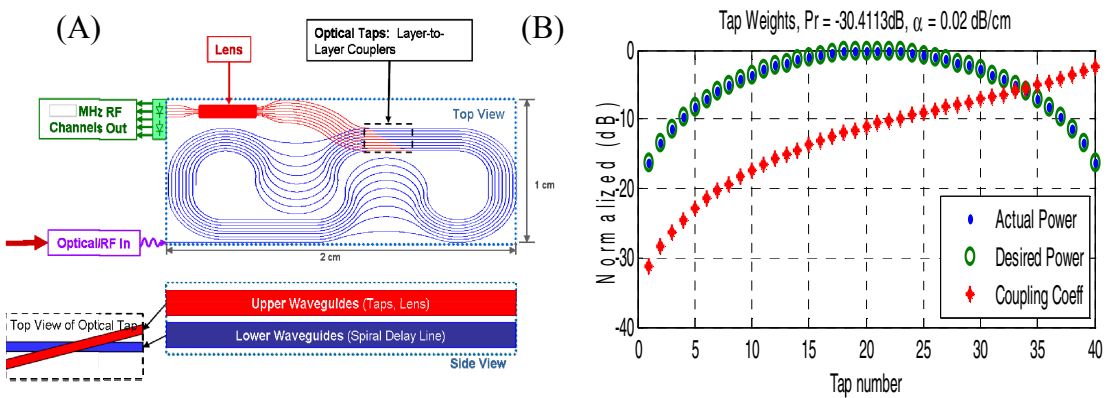

Fig. 1: (A) Schematic diagram of 2 layer tapped delay line design. (B) Taylor windowed tap weights and calculated coupler ratios 


\section{Results}

The two layer $\mathrm{SiO}_{2}$ PICs were fabricated to our design by Enablence USA. The intermediate cladding layer was dimensioned using CMP before deposition of the second waveguide core. Propagation loss in the spiral layer was measured via optical backscatter reflectometer (OBR) as $1.4 \mathrm{~dB} / \mathrm{m}$. We measured the time and frequency domain response of fabricated PICs using the OBR connected to the input and output waveguides by a circulator, which allows direct observation of the delays and tap weights for each lap of the spiral. Data from the central waveguide of the output array is presented, but is characteristic of the other output waveguides. Figure 2(A) shows the measured time domain response for the 40 tap device consistent with the design in figure 1b. The measured frequency response, Figure 2(B), suggests that the phase of each tap has been scrambled by small variations in waveguide geometry and index.

Figure 2(C) shows simulated frequency response using the measured tap weights for random (incoherent) and equalized (coherent) phases. The coherent case reproduces the expected channel pass band $<300 \mathrm{MHz}$, with $25 \mathrm{~dB}$ sidelobe suppression, while the incoherent case is a good qualitative match with measurement.
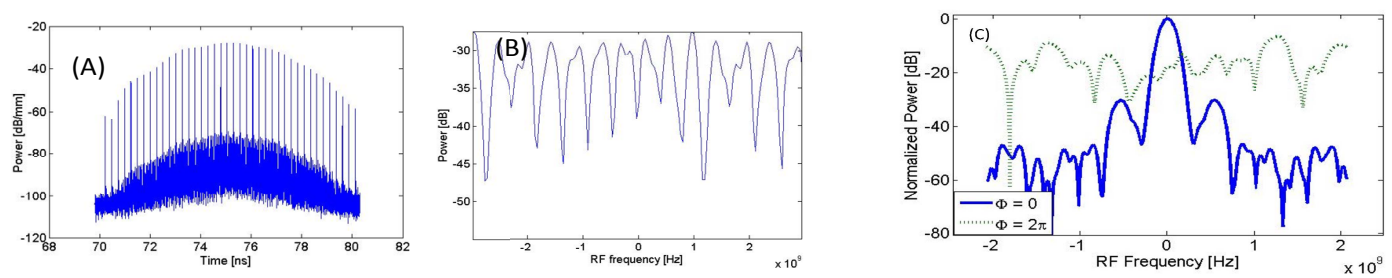

Fig. 2: (A) measured time domain response showing weights of 40 taps (B) measured frequency domain response. (C) Simulated frequency response using tap weights from (A). Taps are assigned a random phase drawn from a uniform distribution [0,Ф].

Takada et al. have demonstrated methods to determine and correct phase errors in AWGs using fiber interferometers [4]. However the stability of these methods is not suitable for the narrow channels of our system. We developed an alternate scheme based on a differential measurement of the filter frequency response using a externally modulated narrow linewidth laser. Power out of a single channel is measured as the RF modulation frequency, $f$, is swept across multiple FSRs. The measured power can be represented as:

$$
I(f)=\left|\Sigma_{m=1 \ldots N}\right| A_{m}\left|\exp \left[j \varphi_{\mathrm{m}}+j 2 \pi f n_{\text {eff }} m \Delta L / c\right]\right|^{2},
$$

where $\varphi_{\mathrm{m}}$ is the phase error of the $m^{\text {th }}$ tap, and $\left|A_{m}\right|$ is its field amplitude. A small phase change, $\Delta \varphi_{1}$, is made to the first tap by UV light exposure and a modified frequency response is measured. Subtracting the two measurements removes all terms with no $\varphi_{1}$ dependence, yielding a difference equation:

$$
\Delta I(f)=j \Delta \varphi_{1} * \Sigma_{m=2 . . N}\left|A_{l}\right|\left|A_{m}\right| \exp \left[j\left(\varphi_{\mathrm{m}}-\varphi_{1}+2 \pi f n_{\text {eff }}(m-1) \Delta L / c\right)\right]+\text { complex conjugate. }
$$

The difference contains $N-1$ sinusoidal terms corresponding to the interference cross term of the $1^{\text {st }}$ and $m^{\text {th }}$ tap. The phase error of the taps relative to the first is extracted using the Fast Fourier Transform of $\Delta I(f)$. Figure 3 shows simulations of the phase measurement at various noise levels, demonstrating phase errors can be accurately determined given sufficient measurement SNR.
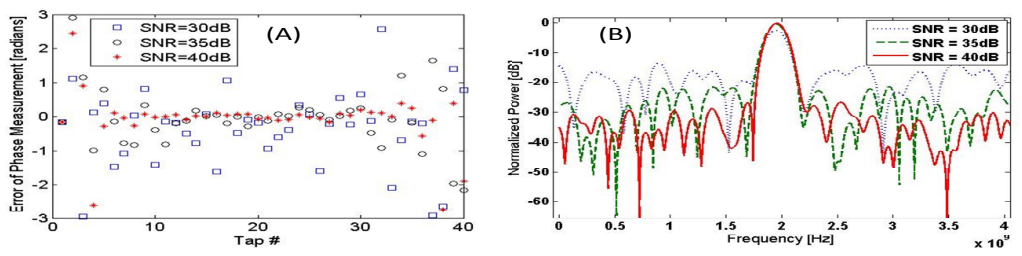

Fig. 3: (A) Simulation of phase error measurement system with SNR of 30,35,40 dB. (B) Simulated filter performance using recovered phases.

\section{Conclusion}

Characterization of the 2 layer PIC filter shows that if phase errors can be accurately compensated, a channelizing filter with RBW of $300 \mathrm{MHz}$ and IBW greater than $4 \mathrm{GHz}$ is achievable. We have developed and simulated a phase error measurement technique suitable for these devices, and are currently implementing the measurement apparatus.

\section{References}

[1] B. Ortega et al., "Advanced Optical Processing of Microwave Signals", EURASIP J. on Applied Signal Processing 10 pp. 1462-1484, 2010.

[2] E.W. Jacobs et al., "RF-over-fiber and Optical Processing for Navy Applications," OFC/NFOEC 2007. pp.1-3, 25-29 March 2007.

[3] M.K Smit and C. van Dam, "PHASAR based WDM-Devices: Principles, Design, and Applications", IEEE J. Sel. Topics Quantum Electron, 2(2), June 1996.

[4] K. Takada and T. Hirose, "Phase-modulation method for AWG phase-error measurement in the frequency domain", Optics Letters 34(24), December 2009. 